\title{
Properties of Neutrinos
}

\section{K. Gabathuler, Villigen}

(Swiss Institute for Nuclear Research)

In the minimal version of the standard theory of unified electromagnetic and weak interactions, neutrinos are strictly massless, neutral particles of spin 1/2. They occur in one helicity state only, viz. left-handed neutrinos $(v)$ and right-handed antineutrinos $\left(v^{c}\right)$. Three different types of neutrinos are known. They are distinguished by assigning to them a property called arbitrarily "flavour". The different flavours are termed according to the charged particles which take part in the creation or absorption of neutrinos: electron $(e)$, muon $(\mu)$, tau $(\tau)$. (There may well be other flavours; however from cosmological arguments, at most one or two additional flavours are expected.) The neutrinos $v_{e^{\prime}}, v_{\mu}, v_{\tau}$ and their charged partners $e, \mu, \tau$ form the class of leptons, which denote particles that are not subject to strong interactions. Flavour conservation leads to the concept of lepton numbers $L_{e^{\prime}} L_{\mu^{\prime}} L_{\tau^{\prime}}$ which are +1 for leptons of the corresponding flavour, -1 for antileptons of the corresponding flavour and 0 otherwise. Each lepton number is conserved in all processes.

These rigorous assumptions of the standard electroweak theory have not been contradicted so far by any confirmed experimental result, but the accuracy of the experimental information is in many cases not very stringent. After having been dormant for some time the interest in neutrino properties has increased greatly with the advent of the less rigorous grand unified theories in which an attempt is made to establish a link between electroweak and strong forces. Based on various large symmetry groups these theories can accommodate neutrino masses in the range between $10^{-6}$ and $10^{+1} \mathrm{eV} / \mathrm{c}^{2}$. Scaling of the neutrino masses with the corresponding charged lepton masses to some unknown power is often expected. In analogy to the quark sector, where mixing occurs among the different quark flavours, neutrino mixing would appear natural. The (physical) flavour states $v_{e}, v_{\mu}, v_{\tau}$ are not necessarily pure neutrino mass eigenstates, but a mixture of the latter. This would give rise to neu- trino oscillations where the flavour composition of a neutrino changes periodically in time. Such an effect requires non-degenerate neutrino mass eigenvalues, i.e. at least one non-zero mass. Thus neutrino oscillations would signal neutrino mass and flavour non-conservation.

The question, whether neutrinos are Dirac particles or Majorana particles, is still outstanding. The only possible mass term for a Dirac particle, described by a spinor $\psi$, is

$$
L_{D}=m_{D} \bar{\psi} \psi
$$

This expression, valid for the charged, massive Dirac particles like the electron, also applies to a massive Dirac neutrino. It conserves the total lepton number $L_{\text {tot }}=L_{e}+L_{\mu}+L_{\tau}$.

Since neutrinos are neutral, another mass term is also allowed:

$$
L_{M}=m_{M}\left(\bar{\psi}^{\mathrm{c}} \psi+\bar{\psi} \psi^{\mathrm{c}}\right)
$$

where $\psi^{\mathrm{c}}$ is the charge conjugated spinor. For charged leptons this Majorana mass term would violate charge conservation. The term $L_{M}$ transforms neutrinos into antineutrinos and vice versa and therefore changes the lepton number $L_{\text {tot }}$ by $\left|\Delta L_{\text {tot }}\right|=2$. For Majorana neutrinos the concept of lepton number is pointless; there is no distinction made between particle and antiparticle. Most grand unified theories predict massive Majorana neutrinos, and lepton number conservation is expected to break down at some level. A particular process that changes the lepton number by $\left|\Delta L_{\text {tot }}\right|=$ 2 is the neutrinoless double $\beta$-decay. Its existence would indicate that the virtual massive neutrino involved in the process is of Majorana type.

Massive neutrinos are of great consequence in astrophysics and cosmology. The cold light neutrino background predicted from the big bang theory could dominate the total mass of the universe, if the neutrinos were heavier than a few $\mathrm{eV} / \mathrm{c}^{2}$. With $\Sigma m_{\mathrm{v}} \cong 55 \mathrm{eV} / \mathrm{c}^{2}$ they would close the universe (the summation extends over all flavours of light stable neutrinos). From the presently observed expansion rate of the universe the neutrino masses are restricted to $\Sigma m_{\mathrm{v}}<$ $200 \mathrm{eV} / \mathrm{c}^{2}$.
Motivated by the dramatic implications a discovery of neutrino mass would have in particle physics, astrophysics and cosmology, many experiments were started in the last decade. Controversial experimental results have stimulated the activity even more. The experiments can be divided into three general classes: Direct kinematical mass measurements, search for neutrino oscillations and search for double $\beta$-decay.

The most persistent claim for neutrino mass comes from a Russian measurement of the electron spectrum near the endpoint in tritium beta decay

$$
{ }^{3} \mathrm{H} \rightarrow{ }^{3} \mathrm{He}+\mathrm{e}^{-}+v_{\mathrm{e}}^{\mathrm{c}} \text {. }
$$

In the Kurie plot of the electron spectrum, a neutrino mass would lead to a departure from a straight line at the very end. The low $Q$-value and the reasonably short lifetime make ${ }^{3} \mathrm{H}$ the most suitable nucleus. A good energy resolution of the electron spectrometer, a very thin, yet luminous tritium source and good background conditions are a prerequisite for a sensitive experiment. The measured electron spectrum is distorted by the finite energy resolution, by the energy loss and backscattering in the source and by atomic and molecular effects governing the transition of the tritium atom (bound in a molecule) into $\mathrm{a}^{3} \mathrm{He}^{+}$ ion. A correct unfolding of these effects is crucial. With a tritiated valine source $\left(\mathrm{C}_{5} \mathrm{H}_{11} \mathrm{NO}_{2}\right)$ the Russian group found a $v_{\mathrm{e}}^{\mathrm{c}}$ mass between 14 and $46 \mathrm{eV} / \mathrm{c}^{2}$. This result, urgently calling for confirmation, triggered a lot of activity in the field. But it took five years until recently the first of the new generation experiments, performed at SIN by a group from the University of Zürich, was able to produce new independent results. The data are consistent with a vanishing $v_{\mathrm{e}}^{\mathrm{c}}$ mass with an upper limit of $18 \mathrm{eV} / \mathrm{c}^{2}$. In this experiment a much simpler source consisting of tritium implanted in carbon was employed. Numerous other experiments with still different tritium sources are now running, so the controversy will be settled rather soon. A sensitivity to the $v_{e}^{c}$ mass between 5 and $10 \mathrm{eV} / \mathrm{c}^{2}$ may be expected.

Present mass limits for $v_{\mu}$ and $v_{\tau}$ are less stringent. From the decay of the pion at rest $\pi^{+} \rightarrow \mu^{+}+v_{\mu}, m_{v \mu}<0.26$ $\mathrm{MeV} / \mathrm{c}^{2}$ was obtained at SIN, and from the decay $\tau^{ \pm} \rightarrow \pi^{ \pm} \pi^{+} \pi^{-} v_{\tau}^{(\mathrm{c})}$ for $m_{\mathrm{v} \tau}$ a mass greater than $70 \mathrm{MeV} / \mathrm{c}^{2}$ was excluded at DESY. If the neutrino masses scale with the squared masses of the associated charged leptons, as some grand unified theories predict, these results are roughly equally relevant in giving the $v_{\mathrm{e}}^{\mathrm{c}}$ limit. In the interpretation of the above data we have assumed that 
the neutrino flavour states are pure mass eigenstates with a well defined mass value.

Numerous kinematic experiments are performed to explore the possibility of an admixture of one or more heavy neutrinos $v_{H}$ to light neutrinos (such as $\left.v_{e}, v_{\mu}\right)$. The word "heavy" represents here the entire mass scale allowed by kinematics, without any theoretical prejudice. Let us assume that $N$ neutrino flavour states $v_{F}$ (heavy and light ones) are superpositions of $N$ mass eigenstates $v$

$$
v_{\mathrm{F}}=\sum_{i=1}^{N} U_{\mathrm{F}, \mathrm{i}} v_{\mathrm{i}}
$$

where $U_{F, i}$ is a unitary mixing matrix. It is known from experiment that the admixture of heavy neutrinos to the known light ones must be very small, i.e. a heavy neutrino $v_{H}$ is predominantly composed of one mass eigenstate j, so that the diagonal matrix element $\left|U_{\mathrm{H}, \mathrm{j}}\right| \cong$ $1 \gg\left|U_{L, j}\right|$, where $L$ denotes the flavours of the light neutrinos. However, in spite of the smallness of $\left|U_{L, j}\right|$, heavy neutrinos might be detectable in the decays $\pi$ or $\mathrm{K} \rightarrow$ ev or $\mu v$. In the charged lepton spectrum they would manifest themselves by an additional peak at some energy below the regular charged lepton peak associated with the light neutrino. In three body decays, such as nuclear $\beta$-decay, a discontinuity in the electron spectrum would appear. Flavour conservation would be broken at the instant of decay and the decay into a charged lepton of flavour $L$ and $a v_{H} \cong v_{j}$ would have a branching ratio proportional to $\left|U_{L, j}\right|^{2}$.

With the exception of one (later con- tradicted) experiment, where in the lower portion of the tritium $\beta$-decay spectrum a discontinuity was found corresponding to $m_{\mathrm{vH}}=17 \mathrm{keV} / \mathrm{c}^{2}$, no heavy neutrino admixture was found. This is also true for experiments that looked for decay products of hypothetical heavy neutrinos, e.g. $v_{\mathrm{H}} \rightarrow \mathrm{v}_{\mathrm{L}}+\mathrm{e}^{+}+$ $\mathrm{e}^{-}$. A large range of mixing strengths and masses is by now excluded.

Flavour mixing among light neutrinos, such as $v_{e}, v_{\mu}$ is governed by $\left|U_{\mathrm{L}, \mathrm{i}}\right|$, where $i$ denotes those mass eigenstates of which the light neutrinos are predominantly composed. This is best studied by searching for neutrino oscillations. Let a neutrino of a given flavour be created with energy $E_{\mathrm{v}}$ by a conventional process. If it is a mixture of mass eigenstates with different mass eigenvalues, these states propagate different-

\section{NORTH-HOLLAND PHYSICS PUBLISHING}

\section{Announcing a new section of Nuclear Physics B: PROCEEDINGS SUPPLEMENTS}

Nuclear Physics B - Proceedings Supplements, will comprise a representative sample of the highest quality Conference Proceedings in the fields central to or in the potential areas of interest of the journal: particle physics, hadronic physics, cosmology. astrophysics, gravitation, field theory and statistical systems.

An international board of associate editors is being appointed with the responsibility to ensure the highest standards for the selection of appropriate conferences and the presentation of their proceedings. The following have already agreed to serve on the board:
S. Brodsky, Stanford, CA, USA

J.-L. Gervais, Paris, France

N. Isgur, Toronto, Canada

C. Llewellyn Smith, Oxford, UK

R. Peccei, Hamburg, FRG

H.R. Rubinstein, Stockholm, Sweden

G. Veneziano, Geneva, Switzerland S. Weinberg, Austin, TX, USA

Further appointments will follow in the near future.

Organizers of upcoming meetings who are interested in exploring the possibilities of NPB(PS) as a publication outlet for their Conference Proceedings, are invited to send full details of the planned conference to:
Dr. Keith Jones, Administrative Editor "Nuclear Physics B"

c/o NORDITA, Blegdamsvej 17

2100 COPENHAGEN, DENMARK

Tel: (1) 3897 18; Telex: 15216 NBIDK

It is intended that the Proceedings Supplements will be published in

"camera-ready" form within three months of

the Publisher's receipt of the final manuscript. Attractive bulk-order arrangements will be offered to the conference organizers.

For 1987 three volumes are announced. Total subscription price: Dfl. 675.00 . If combined with Nuclear Physics, Section B

or Nuclear Physics, Section A and B: Dfl.555.00. (Prices include postage and handling).

\section{Field Theory in Particle Physics}

\section{Volume 1}

\section{by B. de Wit and J. Smith}

1986 xii +494 pages

Hardbound

Price: US \$45.00/Dfl. 125.00

ISBN 0-444-86996-4

Paperback

Price: US \$23.00/Dfl. 75.00

ISBN 0-444-86999-9

\section{Field Theory in Particle Physics} aims at introducing the use of relativistic field theory for describing interactions between elementary particles. The emphasis is on explaining the principal concepts of perturbative field theory, and on demonstrating their application in practical situations, rather than on setting up a complete and rigorous mathematical framework.

The book is intended for experimentalists and beginning theory students. The only prerequisites are that the students should have a knowledge of quantum mechanics and special relativity.

The material is more than enough for a one-semester course. Some of the more advanced parts should also be useful for a wider audience.
The material has been tested extensively in courses and the book is written in a lucid and engaging style.

The authors have taken great care to make their presentation as self-contained as possible by adding several appendices.

Contents: 1. Classical field theory. 2

Feynman diagrams for spinless fields. 3 . Cross sections and decay rates. 4. Particles with spin one. 5. Particles with spin one-half. 6. Cross sections for processes involvin fermions. 7. Quantum corrections. 8 Dimensional regularization and radiative corrections. 9. Asymptotic behaviour. Appendices. 


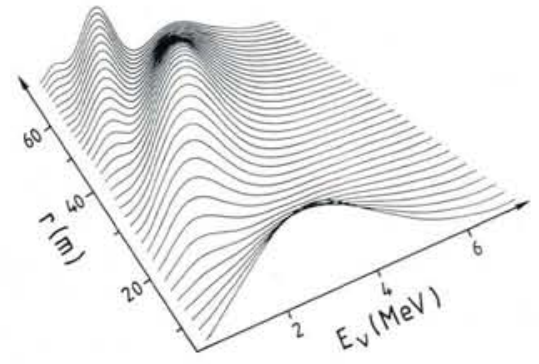

ly in space. This leads to a periodic variation of the flavour composition. For the simplified case where only two flavours are assumed to mix, the wavelength is: $L[m]=2.5 E_{\mathrm{v}}[\mathrm{MeV}] /\left|m_{1}^{2}-m_{2}^{2}\right|\left[\mathrm{eV}^{2} / \mathrm{c}^{4}\right]$, where $m_{1}$ and $m_{2}$ are the eigenvalues of the two mass eigenstates. The amplitude of the oscillation is governed by a mixing angle $\theta$, which parameterizes the two-dimensional mixing matrix

\section{$U=\left(\begin{array}{ll}\cos \theta & \sin \theta \\ -\sin \theta & \cos \theta\end{array}\right)$}

Thus oscillations imply mixing $(\theta \neq 0)$ and non-degenerate mass eigenvalues, i.e. at least one mass must not be vanishing.

Attempts to observe neutrino oscillations have been made by employing neutrinos produced by nuclear reactors or proton accelerators. The detection of neutrinos proceeds via the reaction $v_{\ell}+$ $\mathrm{X} \rightarrow \ell+\mathrm{Y}$, where from a measurement of the charged lepton $\ell$ the neutrino flavour and energy can be deduced. The cross-section for this reaction is extremely small and requires massive detectors or intense neutrino fluxes. A nuclear reactor is a strong source of $v_{e}^{c}$ with energies of a few MeV. With this low energy, new flavours originating from oscillations, such as $v_{\mu}^{c}$, cannot be detected, so one is restricted to measuring the positron spectrum from the reaction $v_{\mathrm{e}}^{\mathrm{c}}+\mathrm{p} \rightarrow \mathrm{e}^{+}+\mathrm{n}$ as a function of distance $r$ from the reactor core. An intensity pattern that does not scale with $1 / r^{2}$ and shows a characteristic energy dependence would signal neutrino oscillations (see Fig. 1). The most comprehensive experiment including measurements at three distances $r$ between 38 $\mathrm{m}$ and $65 \mathrm{~m}$ was performed over a period of four years at the power reactor in Gösgen (Switzerland). The data obtained are consistent with no neutrino oscillations. The mixing and mass parameters are confined to $\left|m_{1}^{2}-m_{2}^{2}\right|<$ $0.019 \mathrm{eV}^{2} / c^{4}$ for maximum mixing $(\theta=$ $\pi / 4)$, and $\sin \theta<0.23$ for $\left|m_{1}^{2}-m_{2}^{2}\right|$ larger than a few $\mathrm{eV}^{2} / \mathrm{c}^{4}$. Furthermore, indications for oscillations found at the reactors in Savannah River (USA) and Le Bugey (France) are contradicted.

Experiments looking for the disappearance of the original flavour, as in the
Fig. 1 - Neutrino oscillations distort the intensity pattern of neutrinos produced with a given flavour, as they travel over a distance $r$. A qualitative example is given for reactor neutrinos detected with a solid angle proportional to $r^{2}$. Two-neutrino flavour oscillations are assumed with maximum mixing $(\theta=\pi / 4)$ and a mass parameter $\left|m_{1}^{2}-m_{2}^{2}\right|=0.2 \mathrm{eV}^{2} / c^{4}$.

case of nuclear reactors, do not depend on any assumption concerning the particular oscillation channel involved, but are quite insensitive to small mixing angles. Appearance experiments, how ever, probe one particular channel, for example $v_{\mu} \rightarrow v_{e}$, by looking for electrons created in a detector exposed to a neutrino beam of originally pure muon flavour. This type of experiment, performed at accelerators at distances of several hundred meters, is quite sensitive to small mixing. The best limit of $\sin \theta<$ 0.028 for $\left|m_{1}^{2}-m_{2}^{2}\right|>10 \mathrm{eV}^{2} / c^{4}$ comes from an experiment at Brookhaven.

The solar neutrino puzzle (the high energy part of the solar $v_{\mathrm{e}}$ flux on Earth is almost three times smaller than expected from the standard solar model) may be explained either by a deficiency of the solar model or by the disappearance of the $v_{e}$ into other flavours. Conventionally the latter interpretation would require almost maximum mixing Only recently has it been realized that because of the different interaction of $v_{e}$ with the electronic matter of the Sun, as compared to the interaction of other neutrinos, the disappearance of $v_{e}$ on their way out of the Sun can be resonantly enhanced, such that mixing angles as small as 0.01 could explain the data. A new radiochemical detector for lower energy solar neutrinos based on $30 \mathrm{t}$ of gallium is now under construction, but the crucial reaction to be explored would be $v+d \rightarrow v+p+n$ using a heavy water detector. This reaction is possible for all neutrino flavours and would allow a definite test of the solar model. Without this test it will be difficult to interpret solar neutrino data in terms of oscillations.

Historically the first kind of oscillations discussed in the literature were oscillations between $v$ and $v^{c}$. This process violates $L_{\text {tot }}$ by $\left|\Delta L_{\text {tot }}\right|=2$ and is further suppressed for helicity reasons, which makes it unattractive.

A more promising $\left|\Delta L_{\text {tot }}\right|=2$ reaction is neutrinoless double $\beta$-decay $(Z, A) \rightarrow$ $(Z+2, A)+2 e^{-}$, where the ordinary decay $(Z, A) \rightarrow(Z+1, A)+e^{-}+v_{e}^{c}$ is energetically forbidden. A neutron $n_{1}$ (see Fig. 2) emits an electron $e_{1}$ and a neutrino. The latter is absorbed by the neutron $\mathrm{n}_{2}$ which then emits the electron $e_{2}$. This process can proceed if the neutrino is a massive Majorana particle.

Neutrinoless double $\beta$-decay has never been observed; however a Majo rana mass of a few $\mathrm{eV} / \mathrm{c}^{2}$ should lead to a measurable effect. Experimentally the most promising case in ${ }^{70} \mathrm{Ge}$ contained in a high resolution, low background $\mathrm{Ge}$ detector. The energy spectrum of the two decay electrons will be a monoenergetic peak at the $Q$-value of $2.04 \mathrm{MeV}$. Large Ge detector arrays with up to 500 $\mathrm{g}^{76} \mathrm{Ge}$ are presently under construction. Detectors and shielding are built from selected material of very low natural radioactivity and will be placed underground, sheltered from cosmic rays. With a measuring time of one year a sensitivity to lifetimes of the order of $10^{24}$ a can be expected. The interpretation of a lifetime or a lifetime limit in terms of a neutrino mass is not straightforward. It involves a knowledge of the second order nuclear matrix element which from calculations is known with an uncertainty of a factor 10 . This translates into a lifetime uncertainty factor of 100. The study of the two-neutrino double $\beta$-decay $(Z, A) \rightarrow(Z+2, A)+2 e^{-}$ $+2 v_{\mathrm{e}}^{\mathrm{c}}$, which is allowed from standard theory, might be useful for a more reliable estimate of the matrix element. Nevertheless, even allowing generously for this uncertainty, Majorana masses well below $10 \mathrm{eV} / \mathrm{c}^{2}$ should be detectable.

\section{Further reading:}

Neutrino Masses and Mixings, by J.L. Vuilleumier,

in Reports on Progress in Physics 49 (1986).

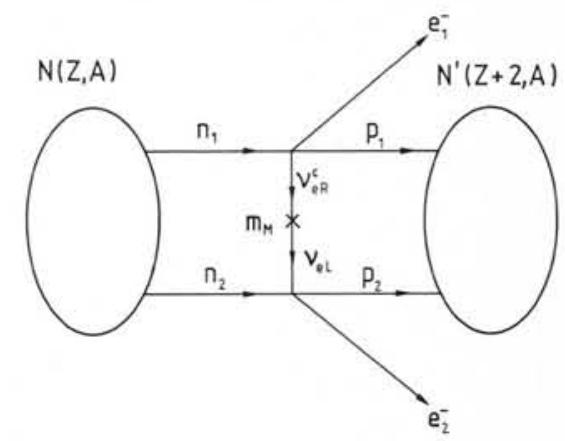

Fig. 2 - Neutrinoless double $\beta$-decay $(Z, A)$ $-(Z+2, A)+2 e^{-}$is not allowed in the standard theory since only purely righthanded antineutrinos can be emitted by the neutron $n$, and only purely left-handed neutrinos can be absorbed by the neutron $n_{2}$ However, a virtual massive Majorana neutrino can be exchanged since Majorana neutrinos are not distinguished from antineutrinos and the Majorana mass $m_{M}$ provides the neutrino with a "wrong" helicity amplitude proportional to $m_{M} / E_{v}$ 